\title{
Molecular detection of influenza A(H1N1)pdm09 viruses with $M$ genes from human pandemic strains among Nigerian pigs, 2013-2015: implications and associated risk factors
}

\author{
O. A. ADEOLA ${ }^{1,2 *}$, B. O. OLUGASA ${ }^{1,3}$ AND B. O. EMIKPE ${ }^{1,4}$ \\ ${ }^{1}$ Centre for Control and Prevention of Zoonoses (CCPZ), University of Ibadan, Ibadan, Nigeria \\ ${ }^{2}$ Department of Medical Microbiology and Parasitology, College of Medicine and Health Sciences, Bingham \\ University, Karu, via Abuja, Nigeria \\ ${ }^{3}$ Department of Veterinary Public Health and Preventive Medicine, Faculty of Veterinary Medicine, University of \\ Ibadan, Ibadan, Nigeria \\ ${ }^{4}$ Department of Veterinary Pathology, Faculty of Veterinary Medicine, University of Ibadan, Ibadan, Nigeria
}

Received 23 May 2017; Final revision 19 September 2017; Accepted 18 October 2017;

first published online 23 November 2017

\section{SUMMARY}

In the post-pandemic period, influenza $\mathrm{A}(\mathrm{H} 1 \mathrm{~N} 1) \mathrm{pdm} 09$ virus has been detected in swine populations in different parts of the world. This study was conducted to determine the presence and spatial patterns of this human pandemic virus among Nigerian pigs and identify associated risk factors. Using a two-stage stratified random sampling method, nasal swab specimens were obtained from pigs in Ibadan, Nigeria during the 2013-2014 and 2014-2015 influenza seasons, and the virus was detected by reverse transcriptase-polymerase chain reaction (RT-PCR). Purified RT-PCR products were sequenced in both directions, and sequences were aligned using MUSCLE. Phylogenetic analysis was conducted in MEGA6. Purely spatial scan statistics and a spatial lag regression model were used to identify spatial clusters and associated risk factors. The virus was detected in both seasons, with an overall prevalence of $8.7 \%$. Phylogenetic analyses revealed that the $\mathrm{M}$ genes were similar to those of pandemic strains which circulated in humans prior to and during the study. Cluster analysis revealed a significant primary spatial cluster $(\mathrm{RR}=4 \cdot 71, \mathrm{LLR}=5 \cdot 66, P=0 \cdot 0046)$, while 'hours spent with pigs $\left(R^{2}=0 \cdot 90, P=0 \cdot 0018\right)$ ' and 'hours spent with pigs from different farms $\left(R^{2}=0.91, P=0.0001\right)$ ' were identified as significant risk factors $(P<0 \cdot 05)$. These findings reveal that there is considerable risk of transmission of the pandemic virus, either directly from pig handlers or through fomites, to swine herds in Ibadan, Nigeria. Active circulation of the virus among Nigerian pigs could enhance its reassortment with endemic swine influenza viruses. Campaigns for adoption of biosecurity measures in West African piggeries and abattoirs should be introduced and sustained in order to prevent the emergence of a new influenza epicentre in the sub-region.

Key words: Human-to-swine transmission, Influenza A(H1N1)pdm09, molecular characterisation, Nigerian pigs, risk factors, spatial pattern.

\footnotetext{
* Author for correspondence: Dr O. A. Adeola, Centre for Control and Prevention of Zoonoses (CCPZ), University of Ibadan, Ibadan, Nigeria.

(Email: phamo2@yahoo.co.nz)
}

\section{INTRODUCTION}

Influenza A viruses cause both natural and experimental infections in humans and animals, and interspecies transmission of these viruses also occurs frequently [1]. Co-infecting influenza viruses could undergo genetic 
reassortment in a permissible host, such as the pig, leading to generation of novel reassortants [2]. In 2009, a novel influenza $\mathrm{A}(\mathrm{H} 1 \mathrm{~N} 1)$ virus, which was to become pandemic, emerged in North America. This virus, later officially referred to as influenza A (H1N1)pdm09 virus (afterward abbreviated as H1N1pdm09 in this paper), was a reassortant with genes from classical swine, North American avian, human seasonal and Eurasian avian-like influenza viruses [3]. In the post-pandemic period, the virus is now known to circulate as a seasonal strain in humans [4].

The virus has also been detected among swine populations in different parts of the world, including North America [5], Europe [6], South America [7], Australia/Oceania [8,9] and Asia [10, 11]. Moreover, in the post-pandemic period, genetic reassortment between $\mathrm{H} 1 \mathrm{~N} 1 \mathrm{pdm} 09$ and other influenza A viruses has been observed to occur especially in pigs [12]. H1N1pdm09 has also been detected among pigs in Africa. For instance, Njabo et al., Ducatez et al. and Adeola et al. provided molecular and/or antigenic evidence of the presence of the 2009 pandemic influenza virus among pigs in Cameroon, Togo and Nigeria, in that order, at various times from 2010 to 2014 [15-17].

Spatial analysis of influenza viruses has been conducted in different parts of the world. For instance, analysis of the 2009 influenza H1N1 pandemic in the United States revealed that the pandemic was characterised by significant spatial heterogeneity. This was because while the 2009 spring wave was found to be restricted mainly to northeastern cities, the larger fall wave affected the whole country [13]. Another study also reported the spatial clustering of swine influenza in Ontario, Canada in 2001 and 2003. In that study, the most likely spatial clusters of cases for each subtype and strain was identified by a spatial scan statistic in a purely spatial Bernoulli model [14]. Since active circulation of the pandemic virus and co-circulation with endemic influenza A viruses in pigs are important for generation of novel reassortants [18], this study was conducted to determine the presence of H1N1pdm09 among pigs in Ibadan, Nigeria and assess the relationship between identified viruses and H1N1pdm09 previously isolated from pigs in Africa. Spatial clusters of H1N1pdm09 infection in the study area were also investigated, and risk factors associated with transmission of the virus at the human-swine interface were identified.

\section{METHODS}

\section{Ethics statement}

This study was approved by the Nigerian National Veterinary Research Institute's Animal Use and Care Committee (NVRIAUCC). In line with The Principles of Humane Experimental Technique, the impact of the procedures on the well-being of animals was minimised by ensuring that specimens were collected by trained personnel early in the morning to reduce heat stress. Moreover, the duration of restraint and handling was minimised. Informed consent was also obtained from each pig handler administered a questionnaire.

\section{Study location}

This study was carried out in Ibadan, the metropolitan capital of Oyo State, Nigeria. The city is located in the South-western geo-political region of the country, between latitude $7^{\circ} 15^{\prime} 00^{\prime \prime} \mathrm{N}$, longitude $3^{\circ} 45^{\prime} 00^{\prime \prime} \mathrm{E}$ and latitude $7^{\circ} 34^{\prime} 00^{\prime \prime} \mathrm{N}$ longitude $4^{\circ} 05^{\prime} 00^{\prime \prime} \mathrm{E}$. It is a prominent transit point between the coastal region and the areas in the hinterland of the country. The city and its environs accounts for 11 of the 18 local government areas (LGAs) in Oyo State, Nigeria [19].

\section{Sampling method and specimen collection}

Collection of swine nasal swab specimens was carried out during the 2013-2014 and 2014-2015 early influenza seasons (December to March). Specimens were collected mostly from adult pigs sampled at the main public abattoir in Ibadan (afterward referred to as Ibadan abattoir) and also from different age groups of pigs in nine piggeries (identified herein as Ibadan farm 1-6 and Akufo farm 1-3) within the study area. These sites were selected based on geographical distribution and representation within Ibadan and environs. At each location, pigs encountered were stratified by age groups, and individual pigs were subsequently selected for sampling by simple randomisation.

Premised on a previous report on the prevalence of influenza in pigs in Ibadan, Nigeria [20], the estimated prevalence was taken to be $14.0 \%$ for Ibadan. At a $95.0 \%$ confidence level, the minimum representative sample size for the study population was calculated, as previously described, to be $185 \cdot 01$ [21]. However, in order to improve the accuracy of results from the study, 218 pigs were used for this study. These pigs 
were either Landrace or Duroc breeds, and they did not show clinical signs of respiratory disease at the time of specimen collection. The proportions of specimens collected for the two influenza seasons, based on categories of pigs, are shown in Fig. 1. In order to enhance their inclusion, younger pigs, which were poorly represented during the first year of the study, were also purposively sampled at three locations (Akufo farm 1, Ibadan farm 3 and Ibadan farm 5) during the 2014-2015 influenza season.

Specimens were collected aseptically as previously described [20] in $2 \mathrm{ml}$ cryo-vial containing virus transport medium. These were immediately transferred into an ice-pack for transport to the laboratory for testing or storage at $-80^{\circ} \mathrm{C}$. Laboratory work was conducted at the Molecular Biology Laboratory of the Centre for Control and Prevention of Zoonoses (CCPZ), University of Ibadan and at the Molecular Biology Laboratory, Bioscience Centre, International Institute of Tropical Agriculture (IITA), Ibadan, Nigeria.

\section{Viral RNA extraction and influenza virus detection}

Viral RNA was extracted from swine respiratory specimens using the ZR Viral RNA Mini Kit (Zymo Research Corporation, Irvine, CA, USA) according to the manufacturer's instructions. Sixty of the specimens collected at Ibadan abattoir, Ibadan farm 1, Ibadan farm 2 and Akufo farm 1 were pooled to reduce diagnostic cost. Pooling was done in groups of 3 (each pool contained $200 \mu$ aliquots from each specimen), based on site of collection, pen in which pigs were located, and age group of pigs, prior to RNA extraction. The concentration and purity of the extracted RNAs were tested using a nanodrop reader (NanoDrop 2000/2000c Spectrophotometer, Thermo Scientific, Wilmington, DE, USA). RNA concentrations were recorded in $\mathrm{ng} / \mu \mathrm{l}$ and purity was read at $260 / 280 \mathrm{~nm}$.

The influenza A type-specific one-step RT-PCR was carried out with forward primer M30F2/08 (5'-ATGAGYCTTYTAACCGAGGTCGAAACG-3') and reverse primer M264R3/08 (5'-TGGACAAANC GTCTACGCTGCAG-3') [22, 23], with One Taq ${ }^{\circledR}$ One-Step RT-PCR Kit (New England BioLabs, Ipswich, MA, USA). The steps involved are summarised as follows. Reagents and samples were taken out from storage and thawed on ice. Reaction components were mixed in sterile RNase-free microfuge tubes to make a $50 \mu \mathrm{l}$ reaction volume. The reaction mix was
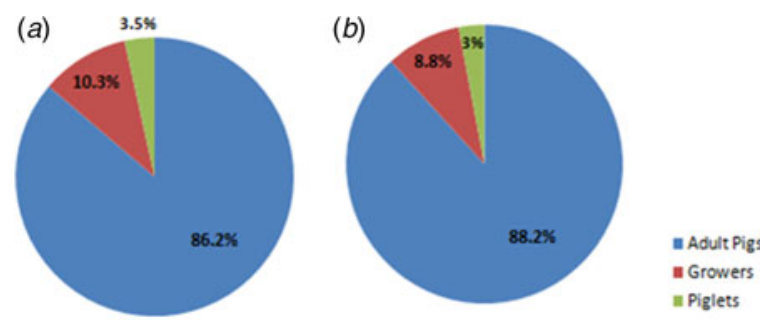

Fig. 1. Pie charts showing proportions of specimens collected from different categories of pigs. (a) Chart for 2013-2014 influenza season. (b) Chart for 2014-2015 influenza season.

prepared by adding $25 \mu 1$ of One Taq One-Step Reaction Mix $(2 \times), 2 \mu \mathrm{l}$ of One Taq One-Step Enzyme Mix $(25 \times), 2 \mu 1$ of Gene-specific Forward Primer $(10 \mu \mathrm{M}), 2 \mu \mathrm{l}$ of Gene-specific Reverse Primer $(10 \mu \mathrm{M})$ and $14 \mu \mathrm{l}$ of Nuclease-free water. To this, $5 \mu \mathrm{l}$ of RNA template was added. Control reactions were also included.

Haemagglutinin (HA) subtyping one-step RT-PCR was carried out with the same RT-PCR Kit and protocol, with forward primer H1F1 (5'-AGCAAAA GCAGGGGAAAATAAAAGC-3') and reverse primer H1R1264 (5'-CCTACTGCTGTGAACTGTGTATTC $-3^{\prime}$ ) for H1N1pdm09 [23, 24]. PCR products were analysed in agarose gel as described by the WHO [23].

\section{Sequencing and phylogenetic analysis}

Positive PCR products representative of the two seasons of this study, and locations from which H1N1pdm09 cases were detected, were purified using ExoSAP (New England BioLabs, Ipswich, MA, USA), according to manufacturer's instructions. Sequencing of the $\mathrm{M}$ gene was done in both forward and reverse directions with the BigDye ${ }^{\circledR}$ Terminator v3.1 Cycle Sequencing Kit (Applied Biosystems, Inc., Foster City, CA, USA) according to manufacturer's instructions on a 3500XL Applied Biosystems capillary sequencer. Sequences were compiled, manually edited and analysed using the Geneious 9.1.4 software (Biomatters Limited, Auckland, New Zealand), and alignment of each sequence was created using MUSCLE [25] provided in the software. Sequence searches were done using the Megablast (highly similar sequences) option of BLAST algorithm (NCBI, Bethesda, MD, USA). Phylogenetic relationships were inferred by NeighborJoining (NJ) algorithm available in MEGA version 6.0 (MEGA6) [26].

In addition to sequences obtained through BLAST searches, all available African swine influenza virus $M$ 
gene sequences (including the only one previously described in Nigeria), $\mathrm{M}$ gene sequence of the prototype H1N1pdm09 strain (A/California/04/2009), and African human H1N1pdm09 M gene sequences from 2013 to 2015 were retrieved from GenBank and included. Some other human H1N1pdm09 M gene sequences which circulated in other countries in 2014 and 2015 were also included to enhance the robustness of the analysis. Evolutionary distances were computed using Maximum Composite Likelihood (MCL) and Kimura-2 methods and are in the units of the number of transitional substitutions per site, and the rate of heterogeneity among sites was modelled as a 4-category discrete gamma distribution. The number of bootstrap replications was set to 1000 . Phylogenetic trees were rooted to the $\mathrm{M}$ gene of $\mathrm{A} /$ chicken/ France/150169a/2015(H5N1).

\section{Nucleotide sequence accession numbers}

Nucleotide sequences of the four viruses from this study are available in GenBank under accession numbers: KX429677-KX429680.

\section{Data}

\section{Case definition}

The case definition was based on recommendations by the World Health Organization (WHO), the World Organization for Animal Health (OIE) and the Food and Agricultural Organization of the United Nations (FAO) [23, 27, 28]. Premised on these recommendations, a sample was considered positive for H1N1pdm09 if results from tests using two different PCR targets (e.g. primers specific for universal $M$ gene and $A(H 1 N 1)$ 2009 HA gene) were positive. Thus, all pigs which tested positive, by RT-PCR, during this study were considered as confirmed cases. Cases were restricted to those from this study because data on confirmatory diagnosis of influenza in pigs in Ibadan and environs were unavailable at relevant government agencies and parastatals. Other H1N1pdm09-negative influenza A viruses detected during this study were also exempted because they did not fit into the case definition.

\section{Focused group discussion}

Prior to this study, information relating to practices within pig farms in the study area was obtained during focused group discussion with consenting members of the Pig Farmers' Association of Nigeria, Oyo State branch, during one of their periodic meetings.
Members of the local branch of this association represented owners of commercial pig farms within Ibadan and environs. Many of these piggery owners had herd sizes ranging from 20 to 100 , and were directly involved in the day-to-day activities of their farms as workers. Large farms, in which more pigs were reared, also existed. Findings from this group discussion were partly used for risk assessment and they aided in the preparation of a questionnaire which was adapted to the study area.

\section{Questionnaire survey}

Enrolled pig handlers were humans who had had regular contact with live pigs, either within commercial pig farms or pig slaughter houses, for at least 6 months prior to the commencement of the study. Administration of the questionnaire to pig handlers was done by trained personnel. Technical terms were avoided as much as possible to ensure that respondents clearly understood the questions. Where such terms had to be used, their meanings were explained to the respondents. Information on routine farm practices, health of pig handler, farm biosecurity, awareness about swine and human influenza, and other relevant details were obtained. Informed consent was obtained from all pig handlers who were administered the questionnaire.

\section{Statistical analyses}

\section{Data from questionnaire}

Data from the questionnaires were collated, and epidemiological and spatial features related to H1N1pdm09 infection at the human-swine interface were identified. Categorical data obtained, using the Likert scale, were processed, as previously described $[29,30]$. For instance, data on 'pig handler's awareness about swine influenza' were obtained on a 4-point Likert scale $(4=$ no knowledge about swine influenza, $3=$ limited knowledge about swine influenza, 2 = substantial knowledge about swine influenza, $1=$ advanced knowledge about swine influenza). For data on 'proximity of pig handler's residence to a pig farm', a 4-point Likert scale was also used $(4=$ lives on pig farm, $3=$ lives within $5 \mathrm{~km}$ of pig farm, $2=$ lives within $10 \mathrm{~km}$ of pig farm, $1=$ lives outside $10 \mathrm{~km}$ of pig farm). Scaling was done, based on answers provided by respondents to relevant questions, by trained personnel who administered the questionnaire.

Descriptive statistics on the seven epidemiological and spatial features were prepared from quantitative 
data and scores from categorical data. In line with previous reports [30-32], these were aggregated to location level and then related to cases of H1N1pdm09 for risk analysis. These features were grouped into two sub-sets of spatial variables. Group 1 comprised features related to degree of proximity and contact between pig handlers and pigs, whereas Group 2 comprised features related to pig handler's health and awareness about influenza.

\section{Purely spatial scan statistic}

High-risk local spatial clusters of H1N1pdm09 infection were detected by Kulldorff's two-dimensional spatial scan statistic [33]. SaTScan software version 9.4.2 [34] was used to run purely spatial scan statistic to determine whether or not the cases were randomly distributed over space in Ibadan and environs. This method is premised on the use of circular window which represents the circular geographical area, and it scans for clusters in space. The scan statistic is advantageous because it controls for covariates and limits pre-selection bias by not specifying a priori observed set of cases within a cluster. In addition, the method does not require a priori knowledge of the population size for elevated risk [32, 33, 35].

In this study, H1N1pdm09 cases were assumed to have a Poisson distribution at each location [36]. Spatial size of scan window was set at $50 \%$ of total population at risk and cluster radius of $10 \mathrm{~km}$. A Monte-Carlo approach with 999 repetitions was performed to test the null hypothesis that there was no difference in relative risk (RR) between H1N1pdm09 clusters. Values of $P<0.05$ were considered to be statistically significant. The cluster with the highest log-likelihood ratio (LLR) and RR was regarded as the cluster least likely to have occurred by chance, while others with significant LLR were considered as secondary clusters $[32,34,36]$.

\section{Classical regression model}

The relationship between H1N1pdm09 cases and seven selected spatial variables was first tested by single variable regression analysis, using the ordinary least squares (OLS) regression model. In the absence of spatial auto-correlation, this model would sufficiently explain the relationship between H1N1pdm09 cases and the spatial variables [31, 32]. However, where spatial auto-correlation exists, the OLS model would lead to biased estimation of parameters and incorrect inference [31, 36].

\section{Spatial lag regression model}

The spatial lag regression (SLR) model, in contrast to spatial error model, integrates the influence of unmeasured independent variables and also stipulates an additional effect of neighbouring attribute values (the lagged-dependent variable). In other words, it integrates spatial effects through inclusion of spatially lagged-dependent variables as predictors. The model was estimated by first creating spatial weights matrix based on the seven spatial features to be tested. This method provides unbiased regression estimates using a Maximum Likelihood approach (ML Spatial Lag) [31, 32].

The seven spatial features identified were sorted into two sub-groups in order to ensure a robust analysis which reveals the effects of characteristics of the two groups separately. Group 1 comprised features related to level of pig handler's proximity to and contact with pigs. Features under this group were (i) proximity of pig handler's residence to a pig farm; (ii) frequency of visit of pig handler to pig pen; (iii) approximate time spent each week with pigs and (iv) approximate time spent each week in other pig farms or with pigs from different farms. Group 2 comprised features related to pig handler's health and awareness about influenza. These were (v) pig handler's awareness about swine influenza; (vi) presence of influenza-like illness among pig handlers and (vii) Handler's knowledge about signs of human influenza.

The regression model was defined by:

$y=\rho W y+X \beta+\varepsilon$

where $y$ is an $N$ defined by $1 \mathrm{H} 1 \mathrm{~N} 1 \mathrm{pdm} 09$ case; $\rho$ is the scalar spatial coefficient; $W y$ is an $N$ defined by 1 weighted matrix of $\mathrm{H} 1 \mathrm{~N} 1 \mathrm{pdm} 09$ cases; $X$ is an $N$ defined by $k$ matrix of explanatory variables; $\beta$ is a $k$ defined by 1 vector of parameters; $\varepsilon$ is an $N$ defined by 1 vector of random error terms [27, 30].

Data analyses were carried out using the Spatial Analyst Software of ArcGIS 10.3 (Environmental Systems Research Institute, Redlands, CA, USA) and GeoDa [37] version 1.8. Values of $P<0.05$ were considered to be statistically significant.

\section{Evaluation of model fitness for identification of risk factors}

A seven symmetric spatial weights matrix, created by the queen contiguity method, was used for Moran's I and Lagrange Multiplier (LM) tests for evaluation of OLS regression residuals for evidence of spatial 
auto-correlation. The OLS and SLR models were subsequently compared using the Akaike information criterion (AIC), Schwarz criterion (SC) and $R^{2}$ value. In line with previous studies, the model with lower $\mathrm{AIC}$ and $\mathrm{SC}$, and higher $R^{2}$ values was considered to have better model fitness [32, 37, 38].

\section{RESULTS}

\section{Molecular detection of H1N1pdm09 infection and phylogenetic analysis}

Twenty-four $(11.0 \%)$ of the 218 swine specimens tested were positive for the presence of influenza A viruses. Nineteen of the 24 influenza A viruses detected were subtyped as H1N1pdm09. More analyses, based on antigen-detection quantitative ELISA and subtyping RT-PCR, were conducted to ascertain the subtypes of the other H1N1pdm09-negative influenza A viruses detected. Their results revealed that the remaining five influenza A viruses detected were of subtype H3N2. During the two influenza seasons of this study, H1N1pdm09 was detected with an overall prevalence of $8.7 \%$. Prevalence in 2013-2014 and 2014-2015 early influenza season was 7.8\% and $9 \cdot 8 \%$, respectively. Figure 2 reveals that adult pigs (boars, sows and gilts), which constituted over $87.0 \%$ of pigs sampled, consistently had the lowest prevalence during the study period. Out of the 10 locations used for the study, H1N1pdm09 was confirmed at three locations. The proportion of cases per positive location is shown in Table 1.

Phylogenetic analysis revealed that the $M$ gene sequences of the strains obtained from Nigerian pigs during this study clustered with sequences of H1N1pdm09 strains which circulated in humans from 2011 to 2013 (Figs 3 and 4). The $\mathrm{M}$ gene sequences of the four Nigerian strains were subsequently compared with the prototype H1N1pdm09 strain (A/California/04/ 2009 FJ969513), with strains previously isolated from pigs in Nigeria [A/swine/Nigeria/12VIR4047-09/2011 (H1N1)] and neighbouring West and Central African countries [A/swine/Togo/ONA32/2013(H1N1) and A/ swine/Cameroon/11rs149-198/2010(H1N1), respectively], and with some closely related human strains. Viruses sequenced in this study grouped with A/Delhi/051/ 2011(H1N1) and A/Delhi/053/2011 (H1N1), and A/ Ghana/KM001/2015(H1N1) with high bootstrap values of 94 and 75, in that order (Fig. 3). Figure 4 (based on the Kimura-2 parameter method) highlights the close relationship between viruses from this study and some human isolates from the same period

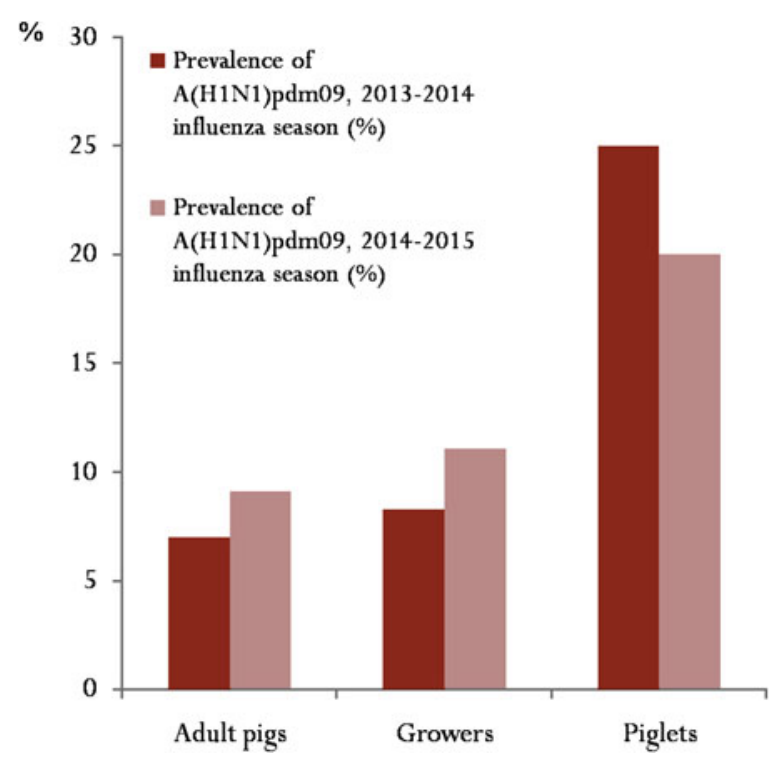

Fig. 2. Prevalence of influenza $A(H 1 N 1) p d m 09$ based on categories of pigs sampled during the study: for this study, adult pigs included boars, sows and gilts.

Table 1. Distribution of cases of influenza A(H1N1) pdm09 per positive location during the study

\begin{tabular}{cllll}
\hline \hline $\begin{array}{c}\text { Positive } \\
\text { locations }\end{array}$ & $\begin{array}{l}\text { Local } \\
\text { government } \\
\text { area }\end{array}$ & $\begin{array}{l}\text { Sample } \\
\text { size }\end{array}$ & $\begin{array}{l}\text { Number } \\
\text { of cases }\end{array}$ & $\begin{array}{l}\text { Detection } \\
\text { rate per } \\
\text { location (\%) }\end{array}$ \\
\hline $\begin{array}{c}\text { Ibadan } \\
\text { abattoir }\end{array}$ & $\begin{array}{c}\text { Ibadan } \\
\text { North }\end{array}$ & 100 & 15 & $15 \cdot 0$ \\
$\begin{array}{c}\text { Ibadan } \\
\text { farm 1 }\end{array}$ & $\begin{array}{c}\text { Ibadan } \\
\text { North }\end{array}$ & 26 & 3 & $11 \cdot 5$ \\
$\begin{array}{c}\text { Akufo } \\
\text { farm 1 }\end{array}$ & Ido & 15 & 1 & $6 \cdot 7$ \\
\hline \hline
\end{tabular}

(2013-2015). Comparative nucleotide sequence analysis of the $\mathrm{M}$ genes of the four viruses sequenced in this study with A/Delhi/051/2011(H1N1), A/Delhi/ 053/2011(H1N1), A/California/04/2009 FJ969513, A/swine/Nigeria/12VIR4047-09/2011(H1N1), A/swine/ Togo/ONA32/2013(H1N1) and A/Ghana/KM001/ 2015(H1N1) is shown in Table 2. Amino-acid differences included non-conservative substitutions in the protein sequence of A/swine/Nigeria/IBDVR004/2015 (H1N1) (S13F) and A/swine/Nigeria/IBDVR004/2015 (H1N1) (A22 T and T37P).

\section{Spatial distribution of H1N1pdm09 cases}

Nineteen cases of swine H1N1pdm09 infection were confirmed during this study, and these occurred mainly in locations within 2 neighbouring LGAs out 


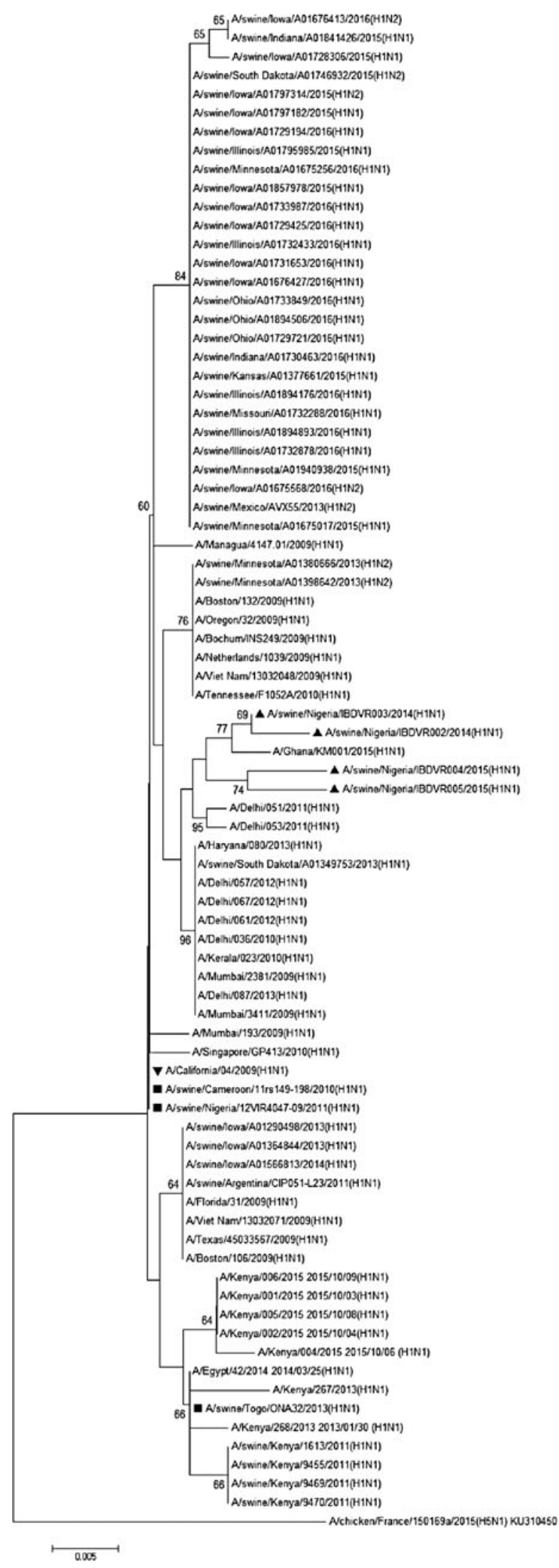

Fig. 3. Phylogenetic analysis of Influenza Matrix Gene Sequences by NJ method (complete tree). Phylogenetic of the 11 LGAs in Ibadan and environs. The locations were Ibadan abattoir and Ibadan farm 1 in Ibadan North LGA and Akufo farm 1 in Ido LGA. A highrisk spatial cluster (hotspot) of swine H1N1pdm09 infection was identified in Ibadan North LGA, Oyo Sate (Ibadan abattoir) (Fig. 5). This significant principal cluster $(P=0.0046)$ had RR of 4.71 , LLR of $5 \cdot 66$, and contained $15(15 \cdot 0 \%, n=100) \mathrm{H} 1 \mathrm{~N} 1 \mathrm{pdm} 09$ cases. A descriptive summary of the primary and secondary purely spatial non-overlapping clusters of H1N1pdm09 cases is provided in Table 3. In all, 18 $(10 \cdot 5 \%, n=171) \mathrm{H} 1 \mathrm{~N} 1 \mathrm{pdm} 09$ cases were confirmed in Ibadan North LGA.

\section{Ordinary least squares regression model}

Based on the results from the OLS regression model, none of the seven spatial features tested was significant as a risk factor for human-to-swine transmission of H1N1pdm09 in Ibadan, Nigeria. A descriptive summary of the classical regression analysis on potential risk factors of $\mathrm{H} 1 \mathrm{~N} 1 \mathrm{pdm} 09$ infection in the study area is presented in Table 4.

\section{Spatial lag regression model}

SLR results revealed that only the Group 1 model was significant, with a likelihood ratio test result of 6.51 $(P=0 \cdot 0107), R^{2}$ value of $0 \cdot 91$, lag coefficient $(R h o)$ of 0.99 and AIC of 42.56, in comparison to Group 2 predictors [likelihood ratio test result of $0.13(P=$ $0 \cdot 7136), R^{2}=0 \cdot 21$, lag coefficient $(R h o)=-0 \cdot 19$ and $\mathrm{AIC}=59 \cdot 83]$. Two important risk factors were identified from this group. These were hours spent each week with pigs and hours spent each week in other

relationship was inferred using the $\mathrm{NJ}$ method. The tree is drawn to scale, and evolutionary distances were computed using the MCL method. The rate variation among sites was modelled with a gamma distribution (shape parameter $=4$ ). Nigerian swine isolates sequenced in this study are indicated by solid triangle $(\boldsymbol{\Delta})$. The prototype H1N1pdm strain (A/California/04/2009) is indicated by inverted solid triangle $(\boldsymbol{\nabla})$. Isolates from neighbouring African countries and a previous isolate from Nigeria are indicated by solid square ( $\mathbf{\square})$. The number of bootstrap replications was set to 1000 , and bootstrap values above 60 are labelled on major tree branches. Evolutionary analyses were conducted in MEGA6 and trees were rooted to A/chicken/France/150169a/2015(H5N1) KU310450. Scale bar indicates the number of nucleotide substitutions per site. 


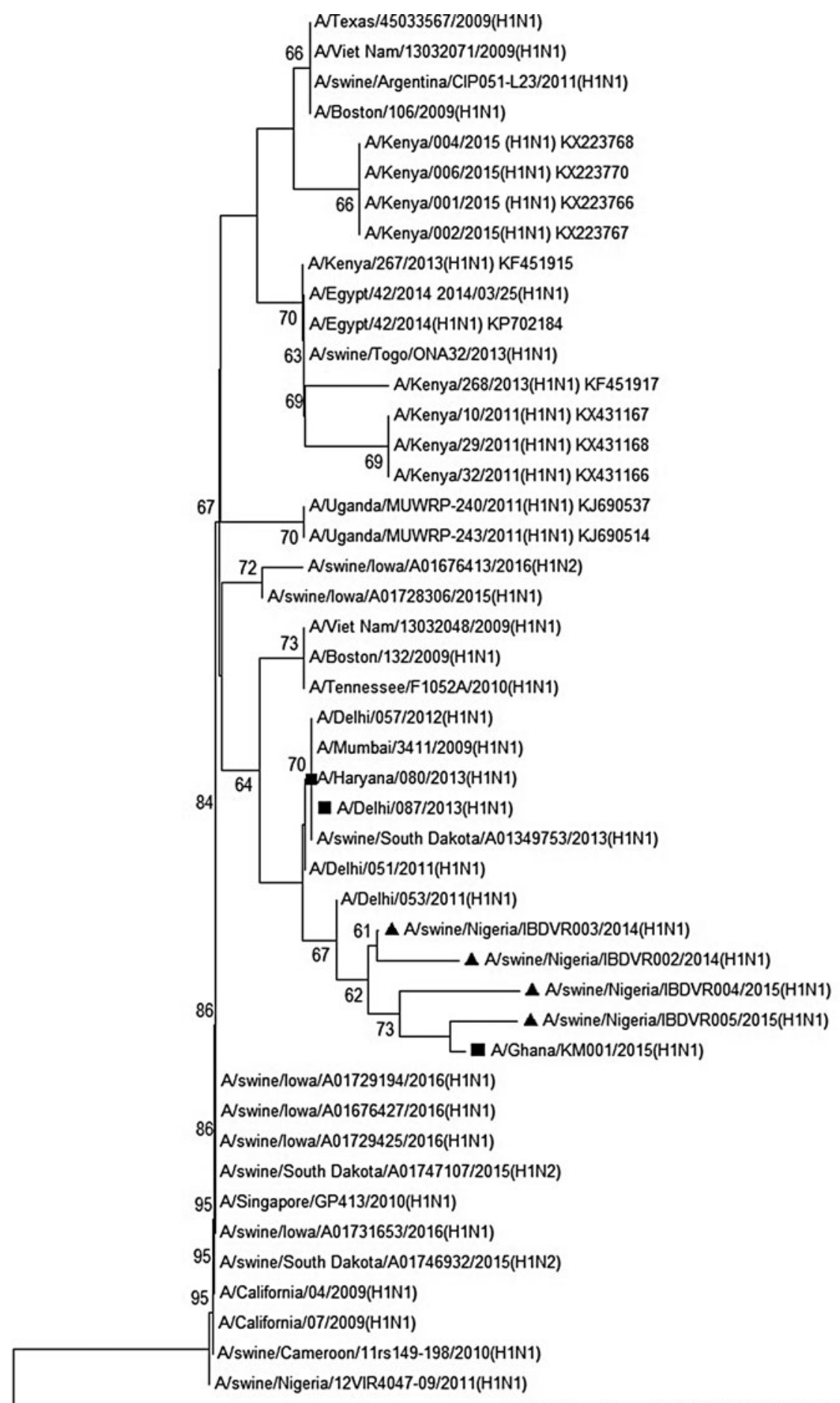

- A/chicken/France/150169a/2015(H5N1) KU310450

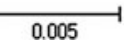

Fig. 4. Relationship between $M$ genes of influenza viruses from this study and some H1N1pdm09 strains which circulated in humans during the period of the study (2013-2015). The tree is drawn to scale, and evolutionary distances were computed using the Kimura- 2 parameter method. The rate variation among sites was modelled with a gamma distribution (shape parameter $=4$ ). Nigerian swine isolates sequenced in this study are indicated by solid triangle $(\mathbf{\Lambda})$, while related

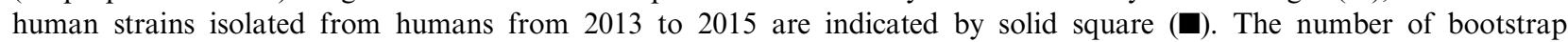
replications was set to 1000 , and bootstrap values above 60 are labelled on major tree branches. Evolutionary analyses were conducted in MEGA6 and trees were rooted to A/chicken/France/150169a/2015(H5N1) KU310450. Scale bar indicates the number of nucleotide substitutions per site. 


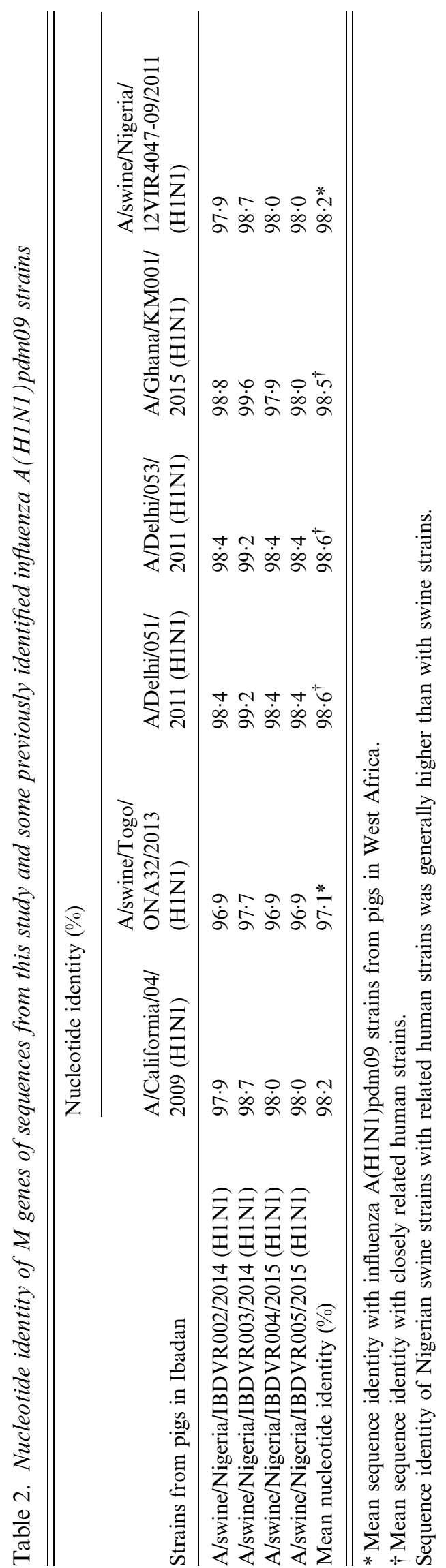

pig farms or with pigs from different farms. A descriptive summary of SLR results for Groups 1 and 2 is provided in Table 5.

\section{Model fitness and risk factors}

Evaluation of OLS regression residuals revealed high level of spatial dependence, with LM (lag) of $9 \cdot 26$ $(P=0 \cdot 00)$ and LM (error) of $1.00(P=0 \cdot 00)$. Robust LM (lag) and Robust LM (error) were 10.00 and $1 \cdot 00$, respectively. AIC and $R^{2}$ values obtained for the OLS model were 52.68 and 0.88 , whereas those estimated for SLR model were 42.56 and 0.91 , in that order. Premised on these findings, which revealed the presence of significant spatial auto-correlation, a spatial lag model was fitted on all the seven spatial features for the identification of risk factors, with Moran's $I=0 \cdot 24$. Thus, two highly significant risk factors (statistical significance was maintained even at $P<0.01$ ), associated with H1N1pdm09 infection among pigs in Ibadan, Nigeria, were identified. These were hours spent each week with pigs $\left(R^{2}=0.90\right.$, $P$-value $=0.0018)$ and hours spent each week in other pig farms or with pigs from different farms $\left(R^{2}=0 \cdot 91, P\right.$-value $\left.=0 \cdot 0001\right)$.

\section{DISCUSSION AND CONCLUSION}

\section{Discussion}

Few cases of human infection with H1N1pdm09 were reported in West and Central Africa, including Nigeria, during the 2009 influenza pandemic. In the post-pandemic period, the virus has been circulating globally as a seasonal human influenza virus [4]. In fact, because of the predominance of the pandemic virus over other seasonal influenza viruses in different countries, the pandemic virus has been consistently recommended as a component of both trivalent and tetravalent influenza vaccines in the last few years [39]. For the 2017-2018 northern and southern hemispheres influenza seasons, A/Michigan/45/2015 (H1N1) pdm09-like virus has been recommended as a component of both trivalent and tetravalent vaccines [40].

During this study, overall prevalence of H1N1pdm09 among pigs in Ibadan $(8.7 \%)$ was lower than those previously reported in the West African sub-region $[16,41]$. Since young, growing pigs are thought to be more susceptible to influenza virus infection [16], the lower prevalence obtained in the present study could be due to the fact that more than $87 \cdot 0 \%$ of pigs tested 


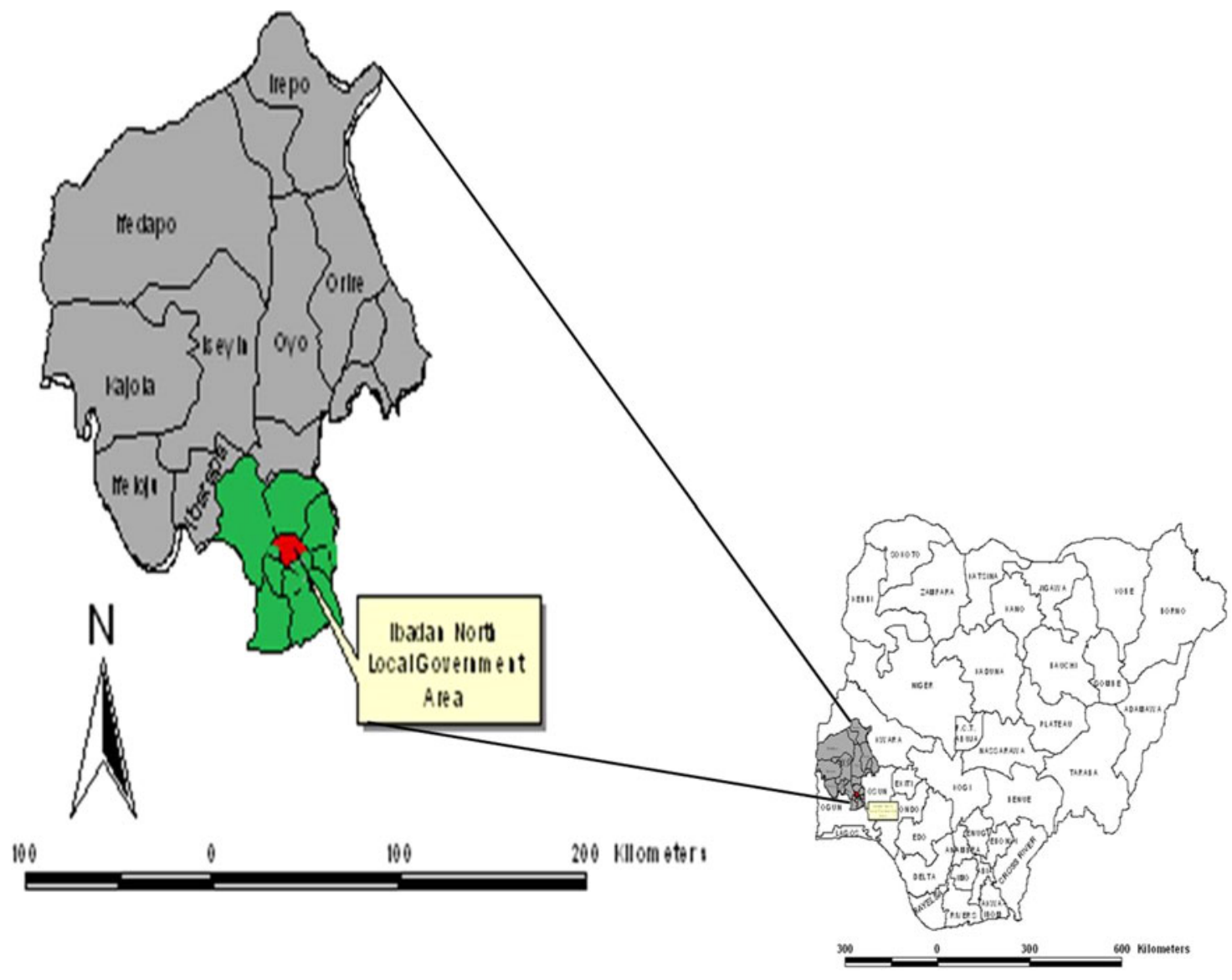

Fig. 5. Spatial pattern of influenza A(H1N1)pdm09 infection among pigs in Ibadan, Nigeria, 2013-2015: (a) Oyo State in south-western Nigeria; $(b)$ enlarged view showing the study area (Ibadan and environs) and the LGA, with clusters of influenza $\mathrm{A}(\mathrm{H} 1 \mathrm{~N} 1)$ pdm09 infection highlighted in green and red, respectively.

Table 3. Purely spatial clusters of influenza A(H1N1)pdm09 infection at location level in Ibadan, Nigeria, 2013-2015

\begin{tabular}{lllllll}
\hline \hline Cluster type & Location & Observed cases* & Expected cases & RR & $P$-value & LLR \\
\hline Most likely (principal) & Ibadan abattoir & 15 & $7 \cdot 82$ & $4 \cdot 71$ & $0 \cdot 0046$ & $5 \cdot 6608$ \\
Secondary & Ibadan farm1 & 3 & $2 \cdot 03$ & $1 \cdot 56$ & 0.9880 & 0.2284 \\
\hline
\end{tabular}

* The case confirmed at Akufo farm 1 was not included in this table.

Table 4. Risk analysis using OLS regression model

\begin{tabular}{llll}
\hline \hline Identified spatial variables* & $B$ & S.E. & Classical regression $p$-value \\
\hline Proximity of handler's residence to pig farm & $1 \cdot 39$ & $1 \cdot 74$ & $0 \cdot 51$ \\
Frequency of visit to pig farm & $-12 \cdot 66$ & $4 \cdot 20$ & $0 \cdot 09$ \\
Hours spent with pigs & $5 \cdot 31$ & $1 \cdot 97$ & $0 \cdot 11$ \\
Hours spent with pigs from different farms & $0 \cdot 39$ & $0 \cdot 81$ & $0 \cdot 68$ \\
Pig handler's awareness about swine influenza & $-0 \cdot 60$ & $0 \cdot 87$ & $0 \cdot 56$ \\
Handler's knowledge about signs of human influenza & $2 \cdot 45$ & $0 \cdot 91$ & $0 \cdot 12$ \\
Presence of influenza-like illness among pig handlers & $-2 \cdot 85$ & $1 \cdot 68$ & $0 \cdot 23$ \\
\hline \hline
\end{tabular}

$B$, beta; s.E., standard error; $P<0 \cdot 05$.

* Potential risk factors of influenza A(H1N1)pdm09 transmission at the human-swine interface. 


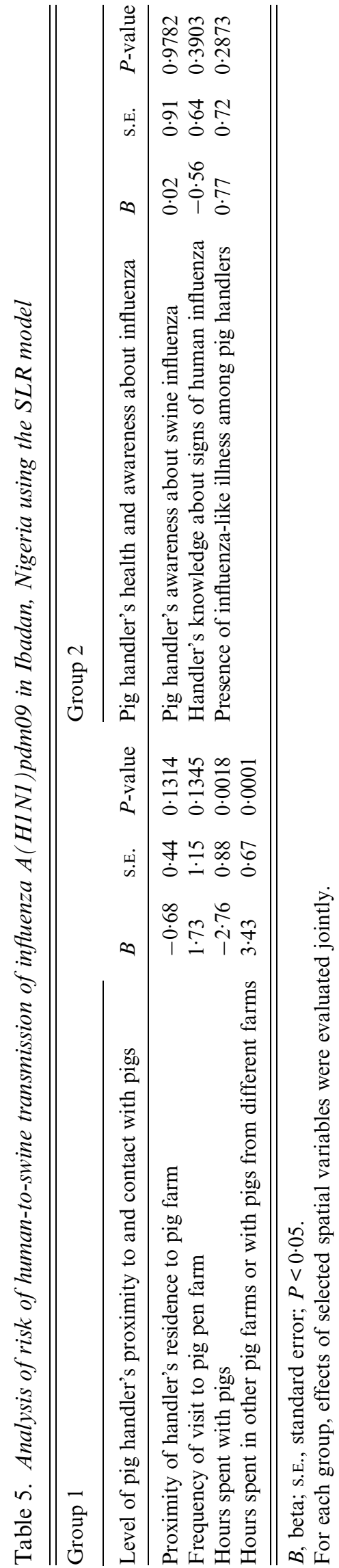

were adult pigs. This was partly because specimens were collected mostly from pigs sampled at an abattoir. In addition, due to some restrictions encountered at some of the pig farms, very few piglets were made available for sampling. Future studies involving detection of influenza viruses in the sub-region should therefore include higher proportion of younger, growing pigs.

As previously noted, pigs sampled during this study did not show clinical signs of respiratory disease at the time of specimen collection. Nonetheless, H1N1pdm09 was observed to have circulated among these pigs during both years of the study. This is also similar to previous results obtained from studies in Nigeria [20, 42], Togo, also in West Africa [16], and Brazil [43] during which influenza viruses were detected among apparently healthy pigs. This underscores the importance of strict adherence to measures which reduce interspecies transmission of influenza viruses at the human-swine interface. These measures include regular hand washing with soap or detergent, enforcement of sick leave for pig handlers (especially during the onset of clinical symptoms of influenza, at which time the shedding of the virus is expected to peak), controlled entrance of visitors to pig pens, provision of farm boots and clothing for handlers and visitors, restriction of handlers to specific units of farm and training of pig handlers on recognition of signs of influenza in humans and pigs [17]. Compliance with these preventive measures should be ensured and monitored, even in the absence of clinical signs of swine influenza.

Minimal genetic variation was observed between the $\mathrm{M}$ genes of viruses detected during the two influenza seasons of this study. Moreover, while S13F substitution has been detected among influenza A viruses in Vietnam [44], the significance of this and other mutations identified in this study has not been clearly elucidated. However, the variations observed in nucleotide and protein sequences of these viruses could be a reflection of the gradual evolution of these viruses, especially through antigenic drift. Sequence and phylogenetic analyses of $M$ genes also revealed that H1N1pdm09 identified among pigs during this study formed a cluster distinct from $\mathrm{H} 1 \mathrm{~N} 1 \mathrm{pdm} 09$ strains previously isolated from pigs in Nigeria, neighbouring West (Togo) and Central (Cameroon) African countries and Kenya in East Africa. On the other hand, the strains identified in the present study clustered with strains of H1N1pdm09 which circulated in humans prior to 
and during this study, from 2011 to 2015. These findings are suggestive of introduction of new strains of H1N1pdm09 into Nigerian swine populations through human-to-swine transmission. They may also be indicative of genetic reassortment between these human strains of the pandemic virus and endemic H1N1 strains circulating in Nigerian pigs. We hope to carry out whole-genome sequencing of these isolates in order to conclusively interpret these findings.

In recent years, new strategies for treatment and prevention of influenza have been developed. However, the risk of interspecies transmission, leading to reassortment of influenza viruses and generation of novel strains, has also increased globally [5-7, 10-12, 45]. Results from the present study revealed the presence of geographical hotspots of swine H1N1pdm09 infection in Ibadan and environs (Fig. 6). Ibadan abattoir was identified as the primary cluster with LLR and RR of 5.66 and 4.71 , respectively. This implies that pigs within this location had $371.0 \%$ increase in risk of infection by the pandemic virus above those within other locations in the study area. In the light of the risk factors identified during this study, the heightened risk of human-to-swine transmission of H1N1pdm09 at this location was due to elevated contact time with pigs. Such closeness between humans and pigs at this abattoir could be due to two key factors.

To start with, pigs transported from different locations were kept, sometimes for up to 3 days, within a lairage located very close to the meat shop. This increased the likelihood of contact between humans (butchers, cleaners, salespersons and even customers) and these pigs. This is similar to the findings of Amorim et al. who reported that continuous exposure of abattoir workers to pigs in Brazil increased the risk for interspecies transmission of influenza A virus [43]. Second, because of very dismal levels of personal and environmental hygiene observed at the abattoir, viruses shed by abattoir workers during the symptomatic phase of influenza could readily infect these pigs. This is corroborated by previous findings which observed that pig handlers in Nigeria had very low levels of compliance with measures which significantly reduce interspecies transmission of influenza [17, 46].

To significantly reduce interspecies transmission of influenza and other diseases at this location therefore, the design of the abattoir should be overhauled to ensure adequate location of the lairage. Pig handlers and other personnel should also be trained on how to maintain adequate personal and environmental hygiene, and compliance with recommended measures should be monitored. Mandatory sick leave for pig handlers is also recommended, especially during the early symptomatic phase of influenza, during which shedding of the virus is expected to peak.

Although infected pigs were mostly slaughtered within 1-3 days after their arrival at the abattoir, the public health significance of H1N1pdm09 infection of these pigs lies in the fact that prolonged exposure of pig handlers to infected pigs at the abattoir could increase the likelihood of swine-to-human transmission of influenza viruses. In addition, some growers brought to the abattoir were occasionally kept close to human dwellings for an extended period of time. Such growing pigs, which have been found to have higher susceptibility to influenza virus infection, could play a role in genetic reassortment and evolution of influenza viruses [10-12, 47]. They may also spread the virus to other pigs or humans. This possibility is even more noteworthy because of occasional foray of extensively raised pigs into the premises of the abattoir.

In rural communities of West Africa, including Nigeria, pigs are commonly reared under extensive (range) system. However, the number of intensive commercial swine production facilities has increased rapidly in recent years, especially in cities such as Ibadan in South-West Nigeria. Many of these farms have inadequate biosecurity measures (which comprises bioexclusion, biomanagement and biocontainment), and pigs are often moved from one farm to another. Many farm hands also worked in two or more pig farms concurrently. This was the situation at Ibadan farm 1, where a secondary cluster of swine H1N1pdm09 infection was identified during this study. Poor adherence to biosecurity measures at this farm must have also contributed to the higher H1N1pdm09 prevalence obtained at this location. The likelihood of contact between humans and pigs at this location was further increased by the fact that these pigs were routinely exposed to undergraduate students from an adjoining tertiary institution. Strict adherence to biosecurity measures is therefore advocated. Such measures include restriction of handlers to specific units of farm, controlled entrance of students and visitors to pig pens, practice of 'all-in-all-out' method and provision of farm boots and clothing for the use of pig handlers, students and visitors [17].

A veiled but very crucial point, which needs to be brought to the fore, is the fact that while the presence of influenza-like illness in piggery workers did not 


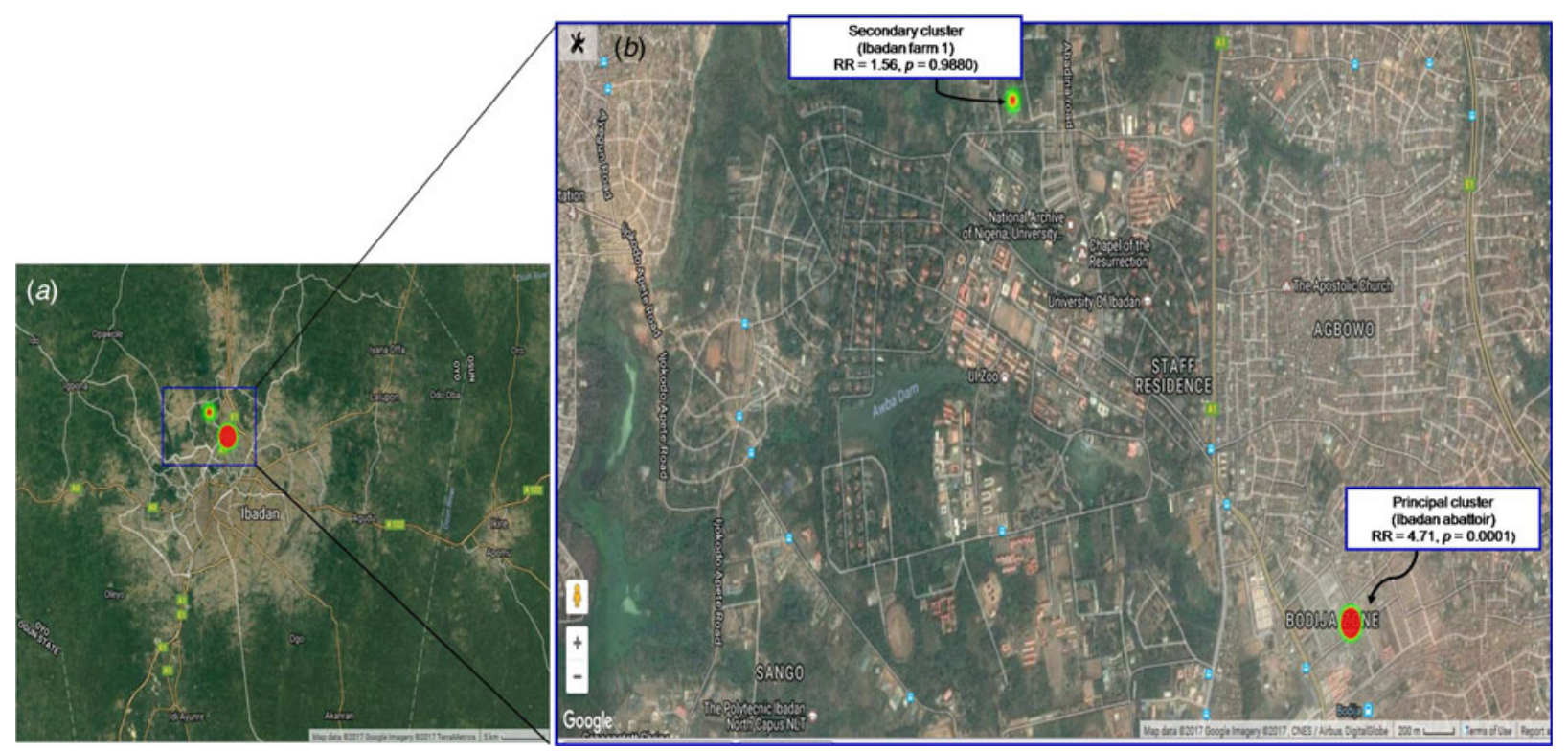

Fig. 6. Heat map of the spatial clusters of influenza A(H1N1)pdm09 infection among pigs in Ibadan, Nigeria, 2013-2015: (a) Ibadan, Oyo State, Nigeria; (b) enlarged view showing locations and cluster intensities at the primary and secondary spatial clusters.

significantly impact influenza detection in pigs, increased contact of piggery workers with pigs from different farms, and transport of pigs from one farm to another, did. This could be a pointer to the important role of fomites in indirect transmission of influenza viruses from humans to pigs, in addition to direct human-to-swine transmission, in the study area. This is in line with previous studies which reported that influenza A viruses, including H1N1pdm09, could survive on fomites for extended periods of time $[48,49]$. In addition, fomites could also be involved in indirect transmission of influenza A viruses from infected to uninfected pigs [50]. Thus apart from introduction of influenza viruses into a piggery through infected pigs brought-in from other farms, pig handlers who work concurrently in different piggeries could inadvertently spread influenza viruses from one farm to the other through fomites, such as farm tools, feed and water troughs and boots. Interestingly though, adequate biosecurity remains effective for the prevention of influenza transmission through any of these routes.

Active circulation of H1N1pdm09 in a swine population has been known to enhance the probability of reassortment with other endemic influenza viruses [18]. Thus, presence and active circulation of the human pandemic strain in Nigerian pigs, due to the risk factors identified during this study, could lead to genetic reassortment between this strain and endemic swine influenza viruses, and generation of novel strains. This pattern of reassortment has been reported in several countries, and they are reported to occur more frequently among isolates from pigs than those from humans $[10-12,47]$. Urgent steps should be taken by relevant government ministries and agencies [51], and other stakeholders in Nigeria (such as the Pig Farmers' Association of Nigeria), to improve on biosecurity of commercial pig farms and abattoirs by harnessing the recommendations from this study. Campaigns for adoption of adequate bioexclusion, biomanagement and biocontainment measures, in pig farms and abattoirs in West Africa, should also be introduced and sustained in order to prevent the emergence of a new influenza epicentre in the sub-region.

\section{Conclusion}

This study has provided molecular evidence of the presence of influenza $\mathrm{A}(\mathrm{H} 1 \mathrm{~N} 1)$ pdm09 infection among pigs in Ibadan, Nigeria during the early influenza seasons of 2013-2014 and 2014-2015. In addition, phylogenetic analysis revealed that $\mathrm{H} 1 \mathrm{~N} 1 \mathrm{pdm} 09$ strains identified in this study formed a cluster different from H1N1pdm09 strains previously isolated from pigs in West Africa. They, however, clustered with H1N1pdm09 strains which circulated in humans just prior to this study, from 2011 to 2013 . These findings are suggestive of either introduction of new strains 
of H1N1pdm09 into Nigerian swine populations through human-to-swine transmission or fomites, or genetic reassortment between H1N1pdm09 and endemic H1N1 strains circulating in Nigerian pigs. We hope to carry out whole-genome sequencing of these isolates in order to conclusively interpret these findings.

Spatial clusters were identified, and pigs within the primary cluster had $371.0 \%$ increase in risk of infection by the pandemic virus above those within other locations in the study area. Risk factors for humanto-swine transmission of the pandemic virus were also identified. These were 'hours spent with pigs from different farms' and 'hours spent with pigs'. In addition to the risk of human-to-swine transmission, the risk factors identified also suggest a role for fomites in transmission of $\mathrm{H} 1 \mathrm{~N} 1 \mathrm{pdm} 09$ to pigs Ibadan, Nigeria. Active circulation of human influenza strains among Nigerian pigs could enhance genetic reassortment with endemic swine influenza viruses and generation of novel strains.

We recommend that targeted sampling of younger pigs should be conducted in Nigeria and other West African countries in order to determine an accurate estimate of prevalence of influenza viruses and enhance analysis of the risk factors for piglet infection. Campaigns for adoption of adequate biosecurity measures, in pig farms and abattoirs in West Africa, should also be introduced and sustained in order to prevent the emergence of a new influenza epicentre in the West African sub-region.

\section{ACKNOWLEDGEMENT}

The authors sincerely appreciate the management and personnel of Molecular Biology Laboratory, Bioscience Centre of the International Institute of Tropical Agriculture (IITA), Ibadan for their co-operation during this study.

\section{FINANCIAL SUPPORT}

Support for this study was received through a grant from the John D. and Catherine T. MacArthur Foundation to the Center for Control and Prevention of Zoonoses (CCPZ), University of Ibadan, Nigeria (Grant number 97944).

\section{CONFLICT OF INTEREST}

None.

\section{REFERENCES}

1. World Health Organization (WHO). Influenza (Seasonal). Fact sheet November, 2016 (http://www. who.int/mediacentre/factsheets/fs211/en/). Accessed 30 March 2017.

2. Zhou NN, et al. Genetic reassortment of avian, swine, and human influenza $A$ viruses in American pigs. Journal of Virology 1999; 73: 8851-8856 (https://www. ncbi.nlm.nih.gov/pmc/articles/PMC112910/http://jvi.asm. org/content/73/10/8851.long). Accessed 30 March 2017.

3. Smith GVD et al. Origins and evolutionary genomics of the 2009 swine-origin H1N1 influenza A epidemic. Nature 2009; 459: 1122-1125. (https://www.ncbi.nlm. nih.gov/pubmed/19516283). Accessed 21 August 2017.

4. World Health Organization (WHO). Standardization of Terminology of the Pandemic A(H1N1)2009 virus. Influenza (http://www.who.int/influenza/gisrs_laboratory/ terminology_ah1n1pdm09/en/). Accessed 31 March 2017.

5. Nelson MI, et al. Continual reintroduction of human pandemic H1N1 influenza A viruses into swine in the United States, 2009 to 2014. Journal of Virology 2015; 89: 6218-6226. (https://www.ncbi.nlm.nih.gov/pmc/articles/PMC4474294/http://jvi.asm.org/content/89/12/6218. long). Accessed 30 March 2017.

6. Simon G, et al. European surveillance network for influenza in pigs: surveillance programs, diagnostic tools and swine influenza virus subtypes identified in 14 European countries from 2010 to 2013. PLoS ONE 2014; 9: e115815. (https://doi.org/101.371/journal.pone. 0115815). Accessed 21 August 2017.

7. Schaefer R, et al. Isolation and characterization of a pandemic H1N1 influenza virus in pigs in Brazil. Pesquisa Veterinária Brasileira 2011; 31: 761-767. (http://www.scielo.br/scielo.php?script=sci_arttext\&pid= S0100-736X2011000900007http://www.nature.com/nature/ journal/v459/n7250/full/nature08182.html). Accessed 31 March 2017.

8. Holyoake PK, et al. The first identified case of pandemic H1N1 influenza in pigs in Australia. Australian Veterinary Journal 2011; 89: 427-431. doi: 10.1111/ j.1751-08132.0110.0844.x. (https://www.ncbi.nlm.nih. gov/pubmed/22008120). Accessed 20 June 2017.

9. Deng Y-M, et al. Transmission of influenza $\mathrm{A}(\mathrm{H} 1 \mathrm{~N} 1)$ 2009 pandemic viruses in Australian swine. Influenza and Other Respiratory Viruses 2012; 6: e42-e47. doi: 10.1111/j.1750-26592.0120.0337.x. (https://www.ncbi. nlm.nih.gov/pmc/articles/PMC4941678/). Accessed 31 March 2017.

10. Hiromoto Y, et al. Isolation of the pandemic (H1N1) 2009 virus and its reassortant with an H3N2 swine influenza virus from healthy weaning pigs in Thailand in 2011. Virus Research 2012; 169: 175-181. (https:// www.ncbi.nlm.nih.gov/pubmed/22906589). Accessed 30 March 2017.

11. Takemae N, et al. Influenza A viruses of swine (IAV-S) in Vietnam from 2010 to 2015: multiple introductions of $\mathrm{A}(\mathrm{H} 1 \mathrm{~N} 1)$ pdm09 viruses into the pig population and diversifying genetic constellations of enzootic IAV-S. Journal of Virology 2017; 91: e01490-16. (http://jvi. 
asm.org/content/91/1/e01490-16.full.pdf). Accessed 21 August 2017.

12. Vijaykrishna D, et al. Reassortment of pandemic H1N1/2009 influenza A virus in swine. Science 2010; 328: 1529. (https://www.ncbi.nlm.nih.gov/pmc/articles/ PMC3569847/). Accessed 28 March 2017.

13. Gog JR, et al. Spatial transmission of 2009 pandemic influenza in the US. PLoS Computational Biology 2014; 10: e1003635. (https://doi.org/101.371/journal. pcbi.1003635). Accessed 21 August 2017.

14. Poljak Z, et al. Spatial clustering of swine influenza in Ontario on the basis of herd-level disease status with different misclassification errors. Preventive Veterinary Medicine 2007; 81: 236-249. (http://www.sciencedirect. com/science/article/pii/S016758770700102X). Accessed 19 August 2017.

15. Njabo KY, et al. Pandemic A/H1N1/2009 influenza virus in swine, Cameroon, 2010. Veterinary Microbiology 2012; 156: 189-192. (https://www.ncbi.nlm.nih.gov/pmc/ articles/PMC3251638/). Accessed 18 September 2016.

16. Ducatez MF, Awoume F, Webby RJ. Influenza A (H1N1)pdm09 virus in pigs, Togo, 2013. Veterinary Microbiology 2015; 177: 201-205. doi: 10.1016/j.vetmic.2015.020.28. (https://www.ncbi.nlm.nih.gov/pmc/ articles/PMC4388795/). Accessed 15 June 2016.

17. Adeola OA, Olugasa BO, Emikpe BO. Detection of pandemic strain of influenza virus (A/H1N1/pdm09) in pigs, West Africa: implications and considerations for prevention of future influenza pandemics at the source. Infection Ecology and Epidemiology 2015; 5: 30227. doi: 10.3402/iee.v5.30227. (http://www.ncbi. nlm.nih.gov/pubmed/26715380https://www.ncbi.nlm. nih.gov/pmc/articles/PMC4695622/). Accessed 14 May 2016.

18. Lycett SJ, et al. Estimating reassortment rates in co-circulating Eurasian swine influenza viruses. Journal of General Virology 2012; 93: 2326-2336. (http://doi.org/101.099/vir.00.44503-0https://www.ncbi. nlm.nih.gov/pmc/articles/PMC3542128/). Accessed 27 September 2016.

19. Ajayi O, et al. Flood Management in an Urban Setting: A Case Study of Ibadan Metropolis. Hydrology for Disaster Management 2012; Special Publication of the Nigerian Association of Hydrological Sciences pg 65-81 (http:// www.journal.unaab.edu.ng/index.php/NAHS/article/ viewFile/914/882). Accessed 21 August 2017.

20. Adeola OA, Adeniji JA, Olugasa BO. Isolation of influenza A viruses from pigs in Ibadan, Nigeria. Veterinaria Italiana 2009; 45: 383-390. (https://www. ncbi.nlm.nih.gov/pubmed/20391401http://www.izs.it/vet_ italiana/2009/45_3/383.pdf). Accessed 2 June 2017.

21. Daniel WW. Biostatisics: A Foundation for Analysis in the Health Sciences, 7th edn. New York: John Wiley \& Sons, 1999; 180-185, 268-270.

22. Eisfeld AJ, Neumann G, Kawaoka Y. Influenza A virus isolation, culture and identification. Nature Protocols. 2014; 9: 2663-2681. doi: 10.1038/nprot.2014.180 (https:// www.ncbi.nlm.nih.gov/pubmed/25321410). Accessed 20 July 2017.
23. World Health Organization (WHO). WHO information for molecular diagnosis of influenza virus-update (http:// www.who.int/influenza/gisrs_laboratory/molecular_ diagnosis_influenza_virus_humans_update_201403rev2015 05.pdf). Accessed 30 March 2017.

24. Lednicky JA, Loeb JC. Detection and isolation of airborne influenza A H3N2 virus using a sioutas personal cascade impactor sampler. Influenza Research and Treatment. 2013, 8 pages. doi: 10.1155/2013/656825 (https://www.ncbi.nlm.nih.gov/pmc/articles/PMC3810 434/). Accessed 21 August 2017.

25. Edgar RC. MUSCLE: multiple sequence alignment with high accuracy and high throughput. Nucleic Acids Research 2004; 32: 1792-1797. (http://dx.doi.org/ 101.093/nar/gkh340https://www.ncbi.nlm.nih.gov/pmc/ articles/PMC390337/). Accessed 23 May 2017.

26. Tamura K, et al. MEGA6: molecular Evolutionary Genetics Analysis version 6.0. Molecular Biology and Evolution 2013; 30: 2725-2729. (https://www.ncbi. nlm.nih.gov/pmc/articles/PMC3840312/https://academic. oup.com/mbe/article-lookup/doi/101.093/molbev/mst197). Accessed 23 May 2017.

27. Food and Agricultural Organization of the United Nations (FAO). FAO guidelines for surveillance of pandemic $\mathrm{H} 1 \mathrm{~N} 1 / 2009$ and other influenza viruses in swine populations (http://www.fao.org/3/a-ak738e.pdf). Accessed 15 August 2017.

28. World Organization for Animal Health (OIE). Manual of diagnostic tests and vaccines for terrestrial animals (http://www.oie.int/en/international-standard-setting/ terrestrial-manual/access-online/). Accessed 15 August 2017.

29. Kamimura A, et al. Perceived concern about the new strain of the influenza and obtaining the vaccine in China, Japan and South Korea. Journal of Infection and Public Health 2017; 10: 251-257. (https://doi.org/ 101.016/j.jiph.20160.60.04). Accessed 30 August 2017.

30. Sullivan GM, Artino AR. Analyzing and interpreting data from Likert-type scales. Journal of Graduate Medical Education 2013; 5: 541-542. doi: 10.4300/ JGME-5-4-18. (https://www.ncbi.nlm.nih.gov/pmc/arti cles/PMC3886444/). Accessed 30 August 2017.

31. Norman G. Likert scales, levels of measurement and the 'laws' of statistics. Advances in Health Sciences Education: Theory and Practice 2010; 15: 625-632.

32. Olugasa BO, et al. The rubber plantation environment and Lassa fever epidemics in Liberia, 2008-2012: a spatial regression. Spatial and Spatio-Temporal Epidemiology 2014; 11: 163-174. doi: 10.1016/j.sste.2014.040.05 (https:// www.ncbi.nlm.nih.gov/pubmed/25457605). Accessed 16 May 2017.

33. Kulldorff M. A spatial scan statistic. Communications in Statistics - Theory and Methods 1997; 26: 14811496. (http://dx.doi.org/101.080/03610929708831995). Accessed 16 May 2017.

34. SaTScan. Software for the Spatial, Temporal, and Space-Time Scan Statistics. Version 9.4 Boston, MA, USA: Information Management Services Inc., 2015. (http://www.satscan.org/). Accessed 16 May 2017. 
35. Kulldorff M, et al. Evaluating cluster alarms: a spacetime scan statistic and brain cancer in Los Alamos. American Journal of Public Health 1998; 88: 1377-1380.

36. Dhewantara P, et al. Space-time scan statistics of 2007-2013 dengue incidence in Cimahi city, Indonesia. Geospatial Health 2015; 10: 373. Published online 2015 Nov 27. doi: http://dx.doi.org/104.081/gh.20153.73. Accessed 21 August 2017.

37. Anselin L, Syabri I, Kho Y. Geoda: an introduction to spatial data analysis. Geographical Analysis 2006; 38: 5-22. doi: 10.1111/j.0016-73632.0050.0671.x.

38. Haworth J, Cheng T. Non-parametric regression for space-time forecasting under missing data. Computers, Environment and Urban Systems 2012; 36: 538-550. (https://doi.org/101.016/j.compenvurbsys.20120.80.05 http://www.sciencedirect.com/science/article/pii/S0198971 512000816). Accessed 16 May 2017.

39. World Health Organization (WHO). Recommended composition of influenza virus vaccines for use in the 2015-2016 northern hemisphere influenza season (http://www.who.int/influenza/vaccines/virus/recommen dations/2015_16_north/en/) Accessed 31312017.

40. World Health Organization (WHO). Recommended composition of influenza virus vaccines for use in the 20172018 northern hemisphere influenza season 2017 (http:// www.who.int/influenza/vaccines/virus/recommendations/ 201703_recommendation.pdf). Accessed 30 March 2017.

41. Meseko CA, Odaibo GN, Olaleye DO. Detection and isolation of 2009 pandemic influenza A/H1N1 virus in commercial piggery, Lagos Nigeria. Veterinary Microbiology 2014; 168: 197-201. (https://www.ncbi. nlm.nih.gov/pubmed/24321146). Accessed 16 May 2017.

42. Adeola OA, Olugasa BO, Emikpe BO. Antigenic detection of human strain of influenza virus A (H3N2) in swine populations at three locations in Nigeria and Ghana during the dry early months of 2014. Zoonose and Public Health 2016; 63: 106-111. (https://www. ncbi.nlm.nih.gov/pubmed/26094828). Accessed 15 December 2016.

43. Amorim AR, et al. Influenza A virus infection of healthy piglets in an abattoir in Brazil: animal-human interface and risk for interspecies transmission. Memórias do Instituto Oswaldo Cruz 2013; 108: 548-553. (http://doi. org/101.590/0074-0276108052013003). Accessed 16 May 2017.

44. Anwar T, Lal SK, Khan AU. Matrix protein 1: a comparative in silico study on different strains of influenza A H5N1 virus. Bioinformation 2006; 1: 253-256. (https://www.ncbi.nlm.nih.gov/pmc/articles/PMC18916 97/). Accessed 14 March 2017.

45. Adeola OA. Treatment of influenza: prospects of post-transcriptional gene silencing through synthetic siRNAs. Exploratory Research and Hypothesis in Medicine 2017; 2: 1-11. DOI: 101.4218/erhm.20160.0013 (https://publinestorage.blob.core.windows.net/77ce760f2454-4dbf-b9e8-e25deff9cfb9/erhm-16-13.pdf). Accessed 8 June 2017.

46. Adeola OA, Adeniji JA. Prevalence of antibodies to influenza viruses among handlers of live pigs at three locations in Ibadan, Nigeria. Veterinaria Italiana 2010; 46: 147-153. (http://www.ncbi.nlm.nih.gov/pubmed/ 20560124http://www.izs.it/vet_italiana/2010/46_2/147. pdf). Accessed 16 May 2017.

47. Ducatez MF, et al. Multiple reassortment between pandemic (H1N1) 2009 and endemic influenza viruses in pigs, United States. Emerging Infectious Diseases 2011; 17: 1624-1629. (https://dx.doi.org/103.201/eid17091. 10338https://wwwnc.cdc.gov/eid/article/17/9/11-0338_ article). Accessed 28 July 2017.

48. Greatorex JS, et al. Survival of influenza $\mathrm{A}(\mathrm{H} 1 \mathrm{~N} 1)$ on materials found in households: implications for infection control. PLoS ONE 2011; 6: e27932. (https://doi. org/101.371/journal.pone.0027932). Accessed 30 August 2017.

49. Oxford J, et al. The survival of influenza $\mathrm{A}(\mathrm{H} 1 \mathrm{~N} 1)$ pdm09 virus on 4 household surfaces. American Journal of Infection Control 2014; 4: 423-425. doi: 10.1016/j.ajic.2013.100.16.

50. Allerson MW, Cardona CJ, Torremorell M. Indirect transmission of influenza A virus between pig populations under two different biosecurity settings. PLoS ONE 2013; 8: e67293. (https://doi.org/101.371/journal. pone.0067293). Accessed 30 August 2017.

51. Vincent A, et al. Review of influenza A virus in swine worldwide: a call for increased surveillance and research. Zoonoses and Public Health, 2014; 61: 4-17. doi: 10.1111/zph.12049. 